\title{
Henry Draper catalogue identifications for Tycho-2 stars st, $^{\star}$
}

\author{
C. Fabricius ${ }^{1}$, V. V. Makarov ${ }^{1,2,3}$, J. Knude ${ }^{1}$, and G. L. Wycoff ${ }^{3}$ \\ 1 Copenhagen University Observatory, Juliane Maries Vej 30, 2100 Copenhagen $\varnothing$, Denmark \\ 2 Universities Space Research Association, 300 D Street S.W., Washington DC 20024, USA \\ 3 United States Naval Observatory, 3450 Massachusetts Ave N.W., Washington DC 20392-5420, USA
}

Received 4 January 2002 / Accepted 14 February 2002

Abstract. We present identifications in the Tycho-2 Catalogue, for 99.8 per cent of the stars in the Henry Draper Catalogue and for 96 per cent of the Henry Draper Extensions.

Key words. stars: fundamental parameters - catalogs

\section{Introduction}

The recently published Tycho-2 Catalogue (Høg et al. $2000)$, provides only cross references to the Tycho- 1 and Hipparcos Catalogues (ESA 1997). HD identifications are widely used and give direct access to the one dimensional spectral classifications in the HD catalogue (Cannon \& Pickering 1918-1924; Cannon 1925-1936; Cannon \& Mayall 1949) and to the two dimensional classifications in the Michigan catalogue (Houk et al. 1975-1999), which presently covers the basic HD stars south of declination $+5^{\circ}$. We have therefore seen a need to facilitate the looking up of HD stars in Tycho-2.

\section{The steps of identification}

For the present work, we have primarily used the machine readable version of the HD Catalogue and its first extension (HDE) prepared by Roman \& Warren (1985) and the version of the Henry Draper Extension Charts (HDEC) prepared by Nesterov et al. (1995). We have corrected various errors in these files as described in the notes.

The HD/HDE gives only very crude positions for the stars, the resolution being only $1^{\prime}-1 \cdot 5$, and as a result identifications are uncertain or even ambiguous for many stars. Although the precision is low, large errors are extremely rare, and we therefore took this piece of astrometric information as the primary indicator of where to look for the matching Tycho-2 star.

\footnotetext{
Send offprint requests to: C. Fabricius, e-mail: cf@astro.ku.dk

* Based on observations made with the ESA Hipparcos astrometry satellite.

** The identifications are available in electronic form at the CDS via anonymous ftp to cdsarc.u-strasbg.fr (130.79.128.5) or via

http://cdsweb.u-strasbg.fr/cgi-bin/qcat?J/A+A/386/709
}

Table 1. The number of stars in various parts of the Henry Draper catalogue, and the final number found in Tycho-2. The section of HDE covering the Large Magellanic Cloud is shown separately.

\begin{tabular}{lrrr}
\hline \hline catalogue & stars & found & rate \\
\hline HD & 225300 & 224869 & 0.998 \\
HDE $\backslash$ LMC & 44950 & 43356 & 0.965 \\
HDE $\cap$ LMC & 1900 & 1459 & 0.768 \\
HDEC & 86933 & 83789 & 0.964 \\
all & 359083 & 353473 & 0.984 \\
\hline
\end{tabular}

The HD positions were transformed to ICRS coordinates, and we then produced a candidate list consisting of all stars from the Tycho-2 Catalogue and its Supplement-1, which, after allowing for proper motion, lie within $3^{\prime}$ of an HD/HDE star or $5^{\prime \prime}$ of an HDEC star and within a very liberal margin of 5 mag. For HD/HDE this gave a single candidate for only half the stars, and up to thirty three candidates for the rest. For HDEC, the much smaller window meant a unique candidate for $94 \%$ of the stars while $4 \%$ were not found in Tycho-2, probably due to the fainter limiting magnitude of HDEC.

In deciding which candidate to prefer, if any, we have been guided by existing cross reference tables; by the Durchmusterung identifications in the HD/HDE; by inspection of the printed HDEC charts; and also by the magnitudes and colours of the candidate stars. Even when there is only one candidate, it can still be the wrong star, and more than one HD star could have the same candidate. Stars completely missing a candidate were checked in existing cross identification tables and a few hundred were recovered in that way.

Due to the large number of stars, it is impossible to make any detailed study of more than a tiny fraction of the cases. The vast majority must be dealt with in an automatic fashion. HD identifications are already included in 

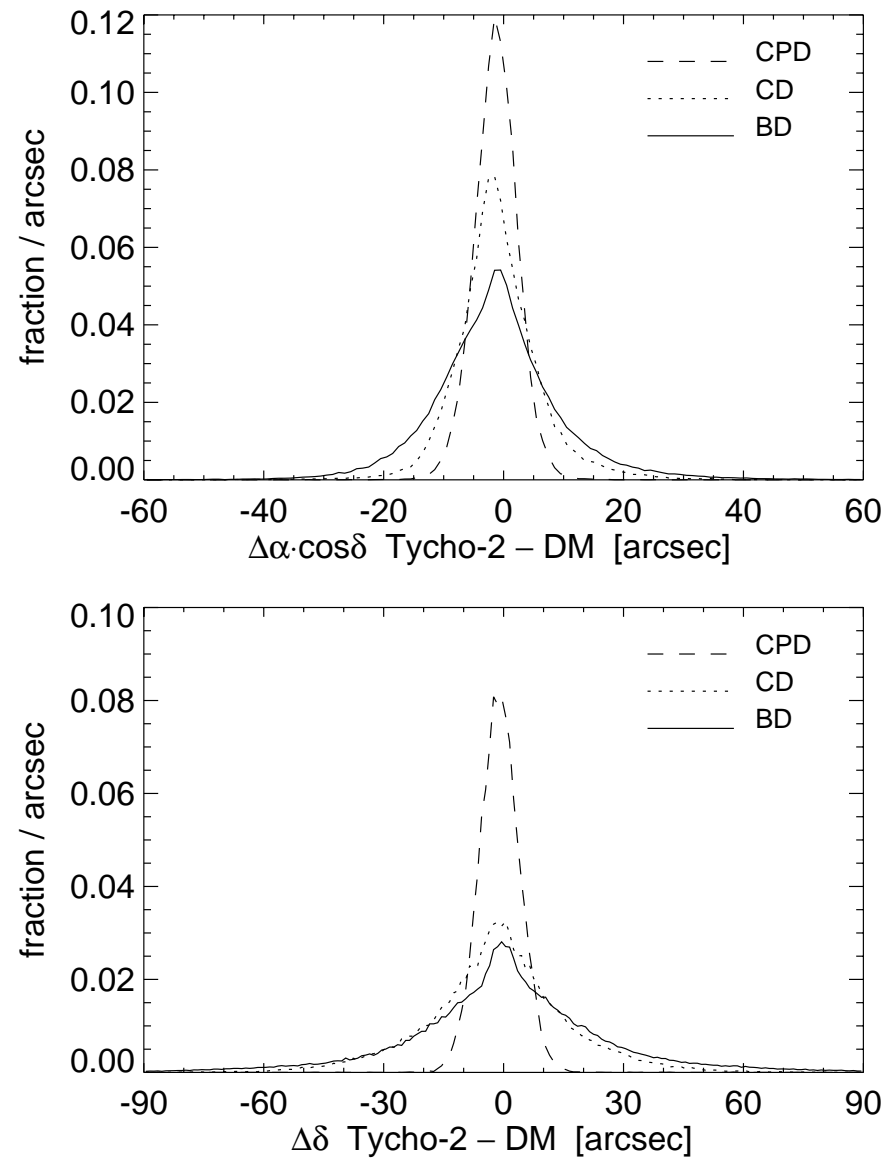

Fig. 1. Positional deviations for the HD stars, between Tycho-2 and the Durchmusterung catalogues. Separate curves show the Bonn (BD), Córdoba(CD), and Cape (CPD) Durchmusterungs.

Tycho-1, HIP and PPM (Röser \& Bastian 1991; Bastian \& Röser 1993). Also, Tycho-1 identifies PPM and HIP stars. From these tables a subset of the HD-Tycho-1 table was extracted, containing no apparent contradictions. These identifications were accepted, provided they were consistent with the candidate list, and did not appear dubious for other reasons. For the remaining cases we normally preferred bright candidates, close to the HD star. Nearly all HD stars, and many HDE stars, have a cross reference to the Durchmusterung (DM) catalogues. The identified Tycho-2 stars were checked for positional agreement, and outliers were investigated. As it can be seen in Fig. 1, the astrometric quality of the DM catalogues is much better than for $\mathrm{HD} / \mathrm{HDE}$, and the DM positions were often used to resolve ambiguous cases. For some HDE stars, identifications are given to the AG (Rogers 1892, Krueger 1890) which also were useful in that respect.

\subsection{The $L M C$ section of the $H D$ extension}

The section of the HDE covering the Large Magellanic Cloud presents a special problem. In the printed volume, the positions for this part, with HD numbers 268601-270500, are given with arcsecond precision, but as $(x, y)$ plate coordinates. In the machine-readable version the positions are given as RA, Dec with an accuracy which, surprisingly, is twice as bad as the rest of the catalogue, leading to numerous problems in this very dense area. From a small set of identified stars, we established the following, simple transformation for obtaining standard coordinates, relative to the tangential point $\left(\alpha_{0}, \delta_{0}\right)=\left(05^{\mathrm{h}} 25^{\mathrm{m}} 12^{\mathrm{s}} .06,-68^{\circ} 54^{\prime} 41^{\prime \prime} 8\right)_{\mathrm{ICRS}}$, from the measured plate coordinates:

$$
\left(\begin{array}{l}
\xi \\
\eta
\end{array}\right)=\left(\begin{array}{rr}
0.9935 & 0.0225 \\
-0.0225 & 0.9935
\end{array}\right)\left(\begin{array}{l}
x-12125 \\
y-10554
\end{array}\right) \frac{\pi}{648000},
$$

from which positions are derived with an accuracy of $5^{\prime \prime}$. We used this tranformation to derive $(x, y)$ coordinates for all the identified Tycho-2 stars, and for the multiple candidates in case of ambiguity, and compared them with the coordinates in the printed volume in order to decide on the identifications.

\section{The identification list}

The identification list gives Tycho-2 identifications for 353473 HD stars, cf. Table 1. For the majority, 352957 stars, it is a one to one identification, while 253 Tycho-2 stars are identified with two HD stars each, and 10 HD stars are resolved in Tycho-2. All these cases are flagged in the list, which also gives the spectral type and a double star flag for stars in the Tycho Double Star Catalogue (Fabricius et al. 2002).

Acknowledgements. This work was supported by the Danish Space Board.

\section{References}

Bastian, U., \& Röser, S. 1993, PPM Star Cat. 3-4 (Heidelberg) Cannon, A. J., \& Pickering, E. C. 1918-1924, Ann. Astron. Obs. Harv. Coll., 91

Cannon, A. J. 1925-1936, Ann. Astron. Obs. Harv. Coll., 100

Cannon, A. J., \& Mayall, M. W. 1949, Ann. Astron. Obs. Harv. Coll., 112

ESA 1997, The Hipparcos and Tycho Catalogues, ESA SP-1200

Fabricius, C., Høg, E., Makarov, V. V., et al. 2002, A\&A, 384, 180

Houk, N., Cowley, A. P., Smith-Moore, M., \& Swift, C. 19751999, Michigan Catalogue of two dimensional spectral types for the HD stars, vols. 1-5 (Ann Arbor, Univ. of Michigan)

Høg, E., Fabricius, C., Makarov, V. V., et al. 2000, A\&A, 363, 385

Krueger, A. 1890, AGK1 zone 55-65, Astron. Gesell. (Leipzig) Nesterov, V. V., Kuzmin, A. V., Ashimbaeva, N. T., et al. 1995, A\&AS, 110, 367

Rogers, W. A. 1892, AGK1 zone 50-55, Astron. Gesell. (Leipzig)

Röser, S., \& Bastian, U. 1991, PPM Star Cat. 1-2 (Heidelberg)

Roman, N. G., \& Warren, W. H. 1985, Documentation for the machine-readable version of the Henry Draper catalogue, NSSDC/WDC-A-R\&S 84-18 